University of Nebraska - Lincoln

DigitalCommons@University of Nebraska - Lincoln

August 1997

\title{
High temperature superconducting Josephson junctions in a stacked bicrystal geometry
}

H.Q. Li

National Institute of Standard and Technology (NIST), Boulder, Colorado

R.H. Ono

National Institute of Standard and Technology (NIST), Boulder, Colorado

L.R. Vale

National Institute of Standard and Technology (NIST), Boulder, Colorado

D.A. Rudman

National Institute of Standard and Technology (NIST), Boulder, Colorado

Sy_Hwang Liou

University of Nebraska-Lincoln, sliou@unl.edu

Follow this and additional works at: https://digitalcommons.unl.edu/physicsliou

Part of the Physics Commons

Li, H.Q.; Ono, R.H.; Vale, L.R.; Rudman, D.A.; and Liou, Sy_Hwang, "High temperature superconducting Josephson junctions in a stacked bicrystal geometry" (1997). Si-Hwang Liou Publications. 64.

https://digitalcommons.unl.edu/physicsliou/64

This Article is brought to you for free and open access by the Research Papers in Physics and Astronomy at DigitalCommons@University of Nebraska - Lincoln. It has been accepted for inclusion in Si-Hwang Liou Publications by an authorized administrator of DigitalCommons@University of Nebraska - Lincoln. 


\title{
High temperature superconducting Josephson junctions in a stacked bicrystal geometry
}

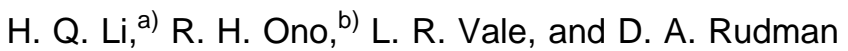 \\ National Institute of Standard and Technology (NIST), Boulder, Colorado 80303 \\ S. H. Liou \\ University of Nebraska-Lincoln, Lincoln, Nebraska 68588-0111
}

(Received 19 May 1997; accepted for publication 19 June 1997)

\begin{abstract}
Bicrystal grain boundary Josephson junctions were fabricated in a stack of two layers of $\mathrm{YBa}_{2} \mathrm{Cu}_{3} \mathrm{O}_{x}$ separated by epitaxial $\mathrm{SrTiO}_{3}$. Weak link behavior was observed in the bridges formed in both layers that had similar shunted-junction characteristics but significantly different critical currents. Characteristic voltages up to $1.9 \mathrm{mV}$ were measured at $4.5 \mathrm{~K}$. The resonant structure was seen in the current-voltage characteristics of the upper-layer junctions, and interactions between junctions in the two layers were evident. (C) 1997 American Institute of Physics.
\end{abstract}

[S0003-6951(97)00534-2]

We have fabricated grain boundary Josephson junctions in a stacked geometry using a multilayer of $\mathrm{YBa}_{2} \mathrm{Cu}_{3} \mathrm{O}_{x}$ (YBCO) and $\mathrm{SrTiO}_{3}$ (STO) on a $24^{\circ}$ STO bicrystal substrate. The fabrication process we developed enables us to test the properties of each layer separately. Both YBCO layers showed weak-link Josephson behavior after patterning into bridge structures that spanned the bicrystal boundary. This is evidence that the grain boundary propagates vertically through the multilayer. The junctions in both layers have similar properties to those observed in single-layer films grown on a bicrystal substrate.

Grain boundary Josephson junctions in high temperature superconducting (HTS) thin films grown on bicrystal substrates $^{1,2}$ have been studied extensively for fundamental science and for many applications. Bicrystal junctions can probe HTS material properties such as the nature of weak links ${ }^{3,4}$ and grain boundary coupling, ${ }^{5}$ penetration depth, and surface resistance. ${ }^{6,7}$ Our experiments allowed us to investigate the propagation of grain boundaries in a multilayer structure. This is an important material science issue itself because many practical devices require multilayer circuits consisting of crossovers, via connections, ${ }^{8,9}$ and stacked superconducting paths over bicrystal grain boundaries. Bicrystal Josephson junctions are promising in device applications such as superconducting quantum interference devices (SQUIDs) $)^{10}$ and voltage standards. ${ }^{11}$ Our stacked grain boundary structure could be used to make devices such as low inductance dc SQUIDs, phase-locked Josephson junctions, and possible three-terminal devices. These potential circuits are possible because the junctions are separated only by the thickness of the insulating film.

Figure 1 is a representation of the wiring scheme of our multilayer test structure showing contacts made independent of each of the YBCO layers containing the desired grain boundary junctions. As in most HTS multilayer circuits, our test samples include crossovers and via connections that require low-angle edges to prevent unwanted grain boundary formation. ${ }^{12}$ We have developed a technique for forming

\footnotetext{
a)Electronic mail: lhanging@central.bldrdoc.gov

b)Electronic mail: ono@boulder.nist.gov
}

such shallow edges using a proximity exposure process suitable for our relatively thick multilayer films. A 2 - $\mu$ m-thick positive photoresist layer is exposed in a contact mask aligner using a multistep procedure. The mask is aligned in contact, then backed off the photoresist by approximately 80 $\mu \mathrm{m}$ and exposed. This results in a defocused image. After development, the photoresist pattern has low-angle edges of about $15^{\circ}$ relative to the film surface. The underlying film is then etched by Ar ion milling, usually at normal incidence. The shallow edges in the photoresist pattern are reproduced in the film; they have a slight difference in angle due to the difference in the ion-milling rates of the photoresist and the film.

The YBCO and STO films were grown by KrF pulsed laser deposition. The YBCO films were deposited at 760$775^{\circ} \mathrm{C}$ with $27 \mathrm{~Pa}(200 \mathrm{mTorr})$ of $\mathrm{O}_{2}$. The STO films were deposited at $740{ }^{\circ} \mathrm{C}$ with $53 \mathrm{~Pa}$ (400 mTorr) of $\mathrm{O}_{2}$. The resistivity of the STO ranged from $2.8 \times 10^{5}$ to 4.6 $\times 10^{7} \Omega \mathrm{cm}$ on several test samples. Typical breakdown voltages ranged from 50 to $100 \mathrm{mV}$ in films $180 \mathrm{~nm}$ thick. We fabricated our junction test sample on a $10 \mathrm{~mm}$

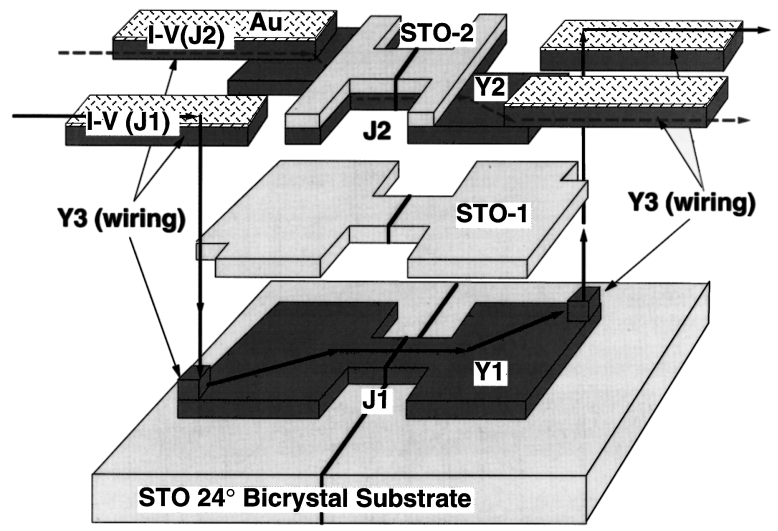

FIG. 1. A schematic drawing of the junction test structure. The drawing is not to scale but instead shows an exploded view of the scheme we used to contact the upper (Y2) and lower (Y1) YBCO layers independently using a single wiring layer $\mathrm{Y} 3$. The solid lines with arrows indicate the direction of current flow used to test junction $\mathrm{J} 1$ in $\mathrm{Y} 1$ and the dashed lines are the path for testing junction $\mathrm{J} 2$ in $\mathrm{Y} 2$. 

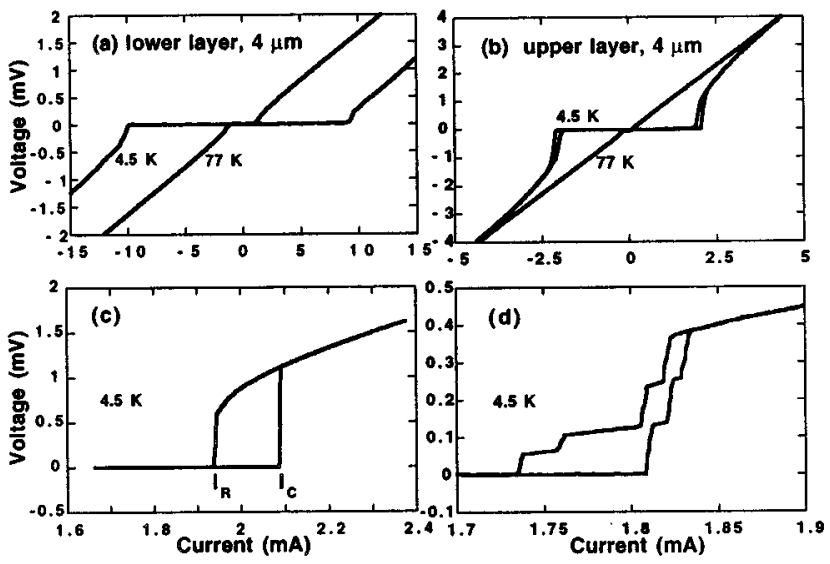

FIG. 2. Current-voltage characteristics of the stacked junctions. Note that the current and voltage scales are different in each of the plots. (a) The $I-V$ curves of junction $\mathrm{J} 1$ in the lower $\mathrm{YBCO}$ layer $\mathrm{Y} 1$ at 4.5 and $77 \mathrm{~K}$. (b) $I-V$ curves at 4.5 and $77 \mathrm{~K}$ for $\mathrm{J} 2$ in the upper YBCO layer Y2. (c) Expanded current scale showing the hysteresis in $\mathrm{J} 2$ at $4.5 \mathrm{~K} . I_{R}$ is the return current and $I_{C}$ is the switching current. (d) Expanded scale showing both hysteresis and self-resonant steps (no external microwaves applied) in another $4 \mu \mathrm{m}$ junction in $\mathrm{Y} 2$ at $4.5 \mathrm{~K}$.

$\times 10 \mathrm{~mm}$ STO bicrystal with a misorientation angle of $24^{\circ}$. We first deposited a four-layer stack composed of $180 \mathrm{~nm}$ YBCO (Y1 in Fig. 1), $180 \mathrm{~nm}$ STO (STO-1), $180 \mathrm{~nm}$ YBCO (Y2), and finally $60 \mathrm{~nm}$ STO (STO-2). The STO-2 layer served as a protecting cap for the layers below and as a seed layer for subsequent epitaxial films. ${ }^{13}$ This four-layer film was patterned as described above to form seven isolated $60 \mu \mathrm{m} \times 100 \mu \mathrm{m}$ rectangles that cross the grain boundary in the substrate and that have low-angle edges. After the ion milling, the photoresist was removed by ultrasonic cleaning in acetone and by oxygen plasma ashing. Another layer of $180 \mathrm{~nm}$ of STO (STO-3, not shown in Fig. 1), was deposited after a final cleaning by low voltage ion milling. This STO layer was used to insulate the exposed edges of $\mathrm{Y} 1$ and $\mathrm{Y} 2$ in the rectangular stacks. The Y2 layer was ion milled to isolate $\mathrm{Y} 1$ as indicated by the corner cutouts in the schematic of Fig. 1. Openings were made through STO-3, STO-2, Y2, and STO- 1 by ion milling at $45^{\circ}$ through a low-angle resist mask. Next, after the removal of photoresist and cleaning of the chip, a third layer of $180 \mathrm{~nm}$ of YBCO and $60 \mathrm{~nm}$ of Au (Y3 in Fig. 1) was deposited and patterned to provide independent superconducting contacts to $\mathrm{Y} 1$ and $\mathrm{Y} 2$ in the stacks. On the four-layer stacks only the openings to Y1 and Y2 were covered by the Y3 layer after patterning by ion milling. After the photoresist was removed, the sample was patterned for the last time to form 4 - and 8 - $\mu \mathrm{m}$-wide microbridges across the grain boundary. At this stage of the process sharp edges are desired on the sides of the bridges so that the two layers have the same cross-sectional area and the junction thickness is approximately uniform across its width.

We will now discuss the results of electrical characterization of the junctions in the two different layers. The current-voltage $(I-V)$ characteristics of this system are rich in features such as hysteresis, resonances, and coupledjunction Shapiro steps. Figure 2(a) shows the $I-V$ characteristics at 4.5 and $77 \mathrm{~K}$ of a $4-\mu \mathrm{m}$-wide junction $\mathrm{J} 1$ in the $\mathrm{Y} 1$ layer. The $I-V$ characteristics of $\mathrm{J} 2$ (in the Y2 layer directly above J1) are shown in Fig. 2(b). Both J1 and J2 showed simple resistively shunted junction (RSJ) behavior ${ }^{14}$ at higher temperatures, as seen in the $77 \mathrm{~K}$ data of Figs. 2(a) and 2(b). The normal resistances are approximately independent of temperature, and no excess current is apparent in the $I-V$ curves. At low temperature their $I-V$ characteristics deviated from the simple RSJ model curves, especially as seen in Fig. 2(a). This can be qualitatively explained in terms of junction width. The simple RSJ model only applies to pointlike junctions with a width $W<4 \lambda_{J}$, where $\lambda_{J}$ $=\left(h / 4 \pi e \mu_{0} J_{C} t\right)^{1 / 2}$ is the Josephson penetration depth ${ }^{14}$ and $t$ is roughly twice the London penetration depth $\lambda_{L}$. Typical values for our YBCO films are $\lambda_{L}=200 \mathrm{~nm}$ at $4.2 \mathrm{~K}$ and 380 $\mathrm{nm}$ at $77 \mathrm{~K}$. We obtain the $\lambda_{J}$ of $\mathrm{J} 1$ to be $0.22 \mu \mathrm{m}$ at $4.5 \mathrm{~K}$ and $0.44 \mu \mathrm{m}$ at $77 \mathrm{~K}$, and the $\lambda_{J}$ of $\mathrm{J} 2$ to be $0.47 \mu \mathrm{m}$ at 4.5 $\mathrm{K}$ and $1.11 \mu \mathrm{m}$ at $77 \mathrm{~K}$. The widths $(4 \mu \mathrm{m})$ of $\mathrm{J} 1$ and $\mathrm{J} 2$ are less than or approximately equal to $4 \lambda_{J}$ at $77 \mathrm{~K}$, but much bigger than $4 \lambda_{J}$ at $4 \mathrm{~K}$. Therefore, we expect deviation from simple RSJ behavior at low temperature; this would be apparent in the microwave and magnetic field response of the junctions.

We observed hysteretic $I-V$ characteristics in both $\mathrm{J} 1$ and $\mathrm{J} 2$ at $4.5 \mathrm{~K}$. This results in part from the relatively large capacitance due to the STO substrate and the STO insulating films. ${ }^{15}$ The expanded scale of Fig. 2(c) shows the hysteretic behavior of $\mathrm{J} 2$ at $4.5 \mathrm{~K}$. The return current $I_{R}$ is $1.89 \mathrm{~mA}$ and the $I_{C}$ is $2.12 \mathrm{~mA}$. From the relation between $I_{R} / I_{C}$ and the McCumber parameter $\beta_{C}=(4 \pi e / h) I_{C} R_{N}^{2} C$ as shown in Ref. 16, we obtain a value of $\beta_{C}=0.9$ for $\mathrm{J} 2$ at $4.5 \mathrm{~K}$. This in turn gives us the capacitance $C$ of $\mathrm{J} 2$ of approximately 2 $\times 10^{-13} \mathrm{~F}$. This inferred capacitance is the parallel combination of the intrinsic capacitance of the junctions and the additional capacitance due to the leads and the STO layers. An accurate estimate of the lead capacitance requires electromagnetic analysis of the four-layer structure. This will be needed to extract an estimate for the relative dielectric constant of the STO and will be the subject of future work.

We observed clear self-resonant steps in the $I-V$ characteristics for a wide temperature range from 4.5 to $60 \mathrm{~K}$ in some of the junctions in the Y2 layer. Figure 2(d) shows the $I-V$ characteristic at $4.5 \mathrm{~K}$ of one junction in the $\mathrm{Y} 2$ layer without external microwave radiation or applied magnetic field (although some trapped flux in the YBCO films may be present which would produce a small dc field). Hysteresis is evident, and we can see a series of current steps at voltages of $60,120,230$, and $360 \mu \mathrm{V}$. These steps are due to resonances in the circuit, with a fundamental frequency corresponding to the $120 \mu \mathrm{V}$ step. The $60 \mu \mathrm{V}$ step then corresponds to a $1 / 2$ harmonic. From the Josephson relation $f$ $=(2 e / h) \mathrm{V}$, we obtain a fundamental frequency of $58 \mathrm{GHz}$. The current step heights vary with temperature, but their voltage positions and therefore the resonance frequencies remain unchanged from 4.5 to $60 \mathrm{~K}$. The resonance frequencies are independent of the junction width; both 4- and 8 - $\mu$ m-wide junctions show a similar structure in their $I-V$ curves. The origin of the resonances is still under investigation. This independence of frequency to junction width probably rules out long junction modes such as zero-field steps and Fiske modes. ${ }^{7,14,17,18}$ The large relative dielectric con- 


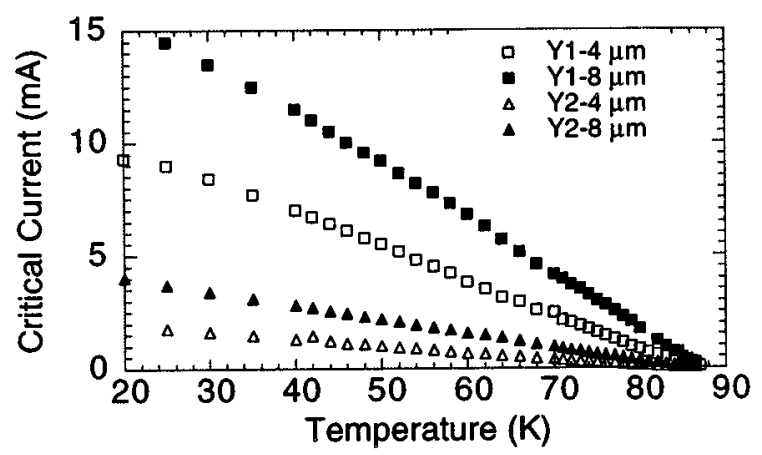

FIG. 3. A family of $I_{C}$ vs $T$ curves: The triangles correspond to a 4 and a 8 $\mu \mathrm{m}$ junction in layer $\mathrm{Y} 2$ and the squares to junctions in layer $\mathrm{Y} 1$. The closed symbols are for the $4-\mu \mathrm{m}$-wide junctions in both layers and the open symbols are the data for the $8-\mu \mathrm{m}$-wide junctions.

stant $\epsilon_{R}$ of the STO makes either a lumped low contraction $\mathrm{LC}$ resonance or a transmission line resonance between YBCO layers a likely candidate as the cause of the observed behavior.

Our test sample is composed of seven stacked junction pairs, four 8 - $\mu \mathrm{m}$-wide junctions, and three 4 - $\mu \mathrm{m}$-wide junctions. The $I_{C}(T)$ dependences of four junctions in two stacked pairs of 4 and $8 \mu \mathrm{m}$ in width are shown in Fig. 3. The square symbols are for the Y1 layer and the triangular symbols for the Y2 layer. The closed symbols are for the 4 $\mu \mathrm{m}$ junctions and the open symbols for $8 \mu \mathrm{m}$ junctions. Junctions in both layers and at both widths showed similar linear $I_{C^{-}} T$ dependence. The critical currents of the Y2 junctions are a factor of 4-6 lower than those of the Y1 junctions directly below them even though the width and thickness of each junction in a stacked pair are the same. A similar reduction in $I_{C}$ was observed in bicrystal grain boundary junctions grown on buffer layers. ${ }^{19}$ The reduction in $I_{C}$ is accompanied by a corresponding increase in $R_{N}$, so the resulting $I_{C} R_{N}$ products are much more similar than either $I_{C}$ or $R_{N}$ alone. At this point, it is not clear whether the lower $I_{C}$ in the Y2 layer is caused by differences in the grain boundaries in the two layers or by a difference observed in the Y2 film quality. We measured a multilayer film grown on a STO substrate (without a bicrystal boundary) under the same conditions as our junction samples and patterned it with same process. The critical current density $J_{C}$ of the second YBCO layer was several times lower than that of the first YBCO layer. We have since determined that by changing the deposition conditions we can reduce the difference in $J_{C}$ to less than a factor of 2 .

We have also observed interactions between junctions in a stack. In this experiment, the junction in Y1 is biased at a fixed current above $I_{C}$, and the Y2 junction is swept; Shapiro steps are evident in the $I-V$ characteristic of the swept junction at the voltage of the Y1 junction. This is presumably due to capacitive coupling of the Josephson oscillations through the STO. Steps were seen up to $2.8 \mathrm{mV}$ at $20 \mathrm{~K}$ and to $0.5 \mathrm{mV}$ at $77 \mathrm{~K}$. This observation implies that the physical proximity of the two junctions in each stack can lead to complex and potentially useful device behavior. A more detailed study of this effect is underway and will be reported elsewhere. This type of sample has allowed us to fabricate compact, very low inductance dc SQUID interferometers.

In conclusion, we report what we believe is the first successful fabrication and testing of bicrystal grain boundary junctions stacked one above the other. Junctions in both layers behave similarly to those reported for grain boundary junctions in a more conventional geometry. The two layers have different $I_{C}$ and $R_{N}$ values (comparing pairs of junctions in stack), but the $I_{C} R_{N}$ product is approximately the same. The difference between Y1 and Y2 junctions therefore may be due to an effectively different cross-sectional area. This junction geometry and the fabrication process can be used to make novel and as-yet untested HTS device structures.

The authors thank John Claassen of the Naval Research Laboratory for the measurements of penetration depth in the YBCO films and acknowledge helpful discussions with Carl Reintsema and Yizi Xu of NIST. One of the authors (S.H.L.) was supported in part by DOE Grant No. DE-FG0290ER45427.

${ }^{1}$ D. Dimos, P. Chaudhari, J. Mannhart, and F. K. LeGoues, Phys. Rev. Lett. 61, 219 (1988).

${ }^{2}$ R. Gross, in Interfaces in Superconducting Systems, edited by S. L. Shinde and D. A. Rudman (Springer, New York, 1992), pp. 176-209.

${ }^{3}$ D. Winkler, Y. M. Zhang, P. A. Nilsson, E. A. Stepantsov, and T. Claeson, Phys. Rev. Lett. 72, 1260 (1994).

${ }^{4}$ D. Dimos, P. Chaudhari, and J. Mannhart, Phys. Rev. B 41, 4038 (1990).

${ }^{5}$ C. L. Lin, W. J. Chang, M. Y. Li, C. H. Li, C. C. Chi, and M. K. Wu, Physica C 269, 291 (1996).

${ }^{6}$ A. Beck, O. M. Froehlich, D. Koelle, R. Gross, H. Sato, and M. Naito, Appl. Phys. Lett. 68, 3341 (1996).

${ }^{7}$ Y. M. Zhang, D. Winkler, G. Brorsson, and T. Claeson, IEEE Trans. Appl. Supercond. 5, 2200 (1995).

${ }^{8}$ F. C. Wellstood, J. J. Kingston, and John Clarke, J. Appl. Phys. 75, 683 (1994).

${ }^{9}$ H. Q. Li, R. H. Ono, L. R. Vale, D. A. Rudman, and S. H. Liou, Appl. Phys. Lett. 69, 2752 (1996).

${ }^{10}$ F. Ludwig, E. Dantsker, R. Kleiner, D. Koelle, J. Clarke, S. Knappe, D. Drung, H. Koch, N. McN. Alford, and T. W. Button, Appl. Phys. Lett. 66, 1418 (1995).

${ }^{11}$ A. M. Klushin, W. Prusseit, K. Urban, E. Sodtke, S. I. Borovitskii, L. E. Amatuni, and H. Kohlstedt, Appl. Phys. Lett. 69, 1634 (1996).

${ }^{12}$ C. L. Jia, K. Kabius, K. Herrman, G. J. Cui, J. Schubert, W. Zander, A. I. Braginski, and C. Heiden, Physica C 175, 545 (1991).

${ }^{13}$ N. Missert, T. E. Harvey, R. H. Ono, and C. D. Reintsema, Appl. Phys. Lett. 63, 1690 (1993).

${ }^{14}$ A. Barone and G. Paterno, Physics and Applications of the Josephson Effect (Wiley, New York, 1982).

${ }^{15}$ Yu. A. Boikov and T. Claeson, J. Appl. Phys. 81, 3232 (1997).

${ }^{16}$ A. B. Zorin, K. K. Likharev, and S. I. Turovets, IEEE Trans. Magn. MAG-19, 629 (1982).

${ }^{17}$ M. D. Fiske, Rev. Mod. Phys. 36, 221 (1964).

${ }^{18}$ T. Doderer, Y. M. Zhang, D. Winkler, and R. Gross, Phys. Rev. B 52, 93 (1995).

${ }^{19}$ A. Beck, A. Stenzel, O. M. Frochlich, R. Gerber, R. Gerdemann, L. Alff, B. Mayer, R. Gross, A. Marx, J. C. Villegier, and H. Moriceau, IEEE Trans. Appl. Supercond. 5, 2192 (1995). 